\title{
CARACTERIZAÇÃO DO ÓLEO E DO FARELO DESENGORDURADO DA POLPA DE MACAÚBA OBTIDOS A PARTIR DA EXTRAÇÃO COM SOLVENTES ORGÂNICOS A BAIXA PRESSÃO
}

\author{
C. P. TRENTINI ${ }^{1}$, D. M. OLIVEIRA ${ }^{2}$, C. M. ZANETTE ${ }^{3}$, C. SILVA $^{1,2}$. \\ ${ }^{1}$ Universidade Estadual de Maringá, Programa de Pós-Graduação em Bioenergia \\ ${ }^{2}$ Universidade Estadual de Maringá, Departamento de Tecnologia \\ ${ }^{3}$ Universidade Estadual do Centro-Oeste, Departamento de Engenharia de Alimentos \\ E-mail para contato: camiladasilva.eq@gmail.com
}

\begin{abstract}
RESUMO - O presente trabalho teve como objetivo a extração do óleo da polpa de macaúba, bem como avaliar as características dos extratos e farelos obtidos. Para este fim, avaliou a influência do tipo de solvente, $n$-hexano, acetato de etila e isopropanol, no rendimento em óleo, perfil de ácidos graxos, teores de $\beta$-caroteno e flavonóides, bem como nas características físico-química e tecnológicas dos farelos desengordurados. Os resultados reportam maiores rendimentos em óleo $(\sim 27,5 \%)$ na utilização do isopropanol $(\mathrm{p}<0,05)$, sendo que os maiores teores em $\beta$-caroteno e flavonóides foram obtidos com este solvente. Na composição dos extratos predomina os ácidos graxos monoinsaturados e relata-se que o tipo de solvente não tem influencia significativa $(p>0,05)$ no perfil de ácidos graxos. $O$ farelo desengordurado apresenta maiores teores de proteína e fibras. A remoção do óleo favorece a capacidade de retenção de água e óleo e volume de intumescimento do farelo, no entanto, não tem influência na capacidade emulsificante.
\end{abstract}

\section{INTRODUÇÃO}

A macaúba (Acrocomia aculeata) apresenta em sua composição óleo de alta qualidade, sendo considerada uma matriz oleaginosa com grande potencial para a produção sustentável de alimentos e biocombustíveis (Silva e Andrade, 2013). A macaúba apresenta melhores condições de rendimento em terras de cerrado, praticamente não sofre ataque de doenças e apresenta elevado teor de óleo ( $30 \%$ ) (Abreu et al., 2011). A polpa da macaúba representa em torno de $45 \%$ da composição do fruto (Ramos et al., 2008) e fornece um óleo com coloração amarelo-alaranjado, rico em compostos bioativos, e na composição de ácidos graxos, elevados teores de ácido oleico e palmítico (Hiane et al., 2005; Ramos et al. 2008; Ciconini et al., 2010; Amaral et al., 2011; Coimbra e Jorge, 2011 a, b). Dentre os compostos bioativos, presente na polpa da macaúba destaca-se o $\beta$-caroteno, um poderoso antioxidante, que é um precursor da vitamina A no metabolismo humano e animal, e os flavonóides que pertencem ao grupo dos compostos fenólicos, destacando-se por sua atividade antioxidante e diversos efeitos biológicos (Prior e Cao, 2000; Huber e Rodriguez-Amaya, 2008). O óleo obtido na polpa da macaúba pode ser usado para diferentes fins industriais, tais como cosméticos, produtos farmacêuticos, alimentos e na produção de biocombustíveis (Amaral et al., 2011; Coimbra e Jorge, 2011a, b; Ciconini et al., 2013).

Entre os métodos para extração de óleo de sementes oleaginosas podemos citar a extração por prensagem, extração com solvente, extração com fluido supercrítico e extração assistida por ultrassom, sendo as duas primeiras consideradas como técnicas convencionais. Recentemente, estudos vêm sendo desenvolvidos como alternativas a estes métodos tradicionais utilizando 
solventes aceitáveis na indústria de alimentos tais como: acetato de etila (Aquino et al., 2009; Almeida et al., 2012; Tian et al., 2013), etanol (Freitas et al., 2007; Vatai et al., 2009; Dutta et al., 2014), isopropanol (Seth et al., 2007; Dutta et al., 2014; Ramluckan et al., 2014) entre outros. Dentre as técnicas aplicadas utilizando tais solventes, os trabalhos de Oliveira et al. (2013a) e Oliveira et al. (2014) reportam a extração sólido-líquido, a baixa pressão, em equipamento de agitação orbital. Em relação à obtenção do óleo da polpa de macaúba, na literatura, reporta-se a utilização da extração por solvente orgânico em Soxhlet (Hiane et al., 2005; Ramos et al., 2008; Coimbra e Jorge, $2011 \mathrm{a}$, b; Coimbra e Jorge, 2012; Ciconini et al., 2013; Oliveira et al., 2013b) e prensagem (Silva e Andrade, 2013).

A obtenção do óleo de macaúba gera o farelo desengordurado como subproduto, o qual pode ser destinado à ração animal e utilizado em formulações alimentícias, como farinha desengordurada após a moagem e peneiramento. A remoção do óleo pode potencializar características tecnológicas do farelo devido ao aumento dos teores de proteína e fibras alimentares, podendo ser utilizado no enriquecimento proteico de produtos e a determinação destas características torna-se essencial a fim estabelecer o potencial de incorporação deste subproduto.

De acordo com o contexto descrito, o presente trabalho teve como objetivo a avaliação de solventes para extração do óleo da polpa de macaúba a baixa pressão. Os experimentos foram conduzidos em equipamento de agitação orbital utilizando razão sólido:solvente de 1:8, mantendo a temperatura em $40^{\circ} \mathrm{C}$, agitação em $40 \mathrm{rpm}$ e tempo de extração em 4 horas. O rendimento em óleo, perfil de ácidos graxos, teores de $\beta$-caroteno e flavonóides, bem como as características físico-química e tecnológicas dos farelos desengordurados são reportadas.

\section{MATÉRIAS E MÉTODOS}

\subsection{Materiais}

Os frutos de Macaúba (Acrocomia aculeata) foram coletados na região do Cariri no Ceará. Os frutos foram higienizados e posteriormente realizou-se a separação das partes: casca, polpa (mesocarpo), endocarpo e amêndoa. A polpa foi submetida à secagem a $60^{\circ} \mathrm{C}$ por $8 \mathrm{~h}$, sendo a umidade determinada em 3,6 $\pm 0,1 \%$. A polpa foi triturada utilizando um moinho elétrico (Marconi) e classificada usando peneiras da série Tyler (Bertel, ASTM) para produzir partículas com diâmetro médio de $0,5 \mathrm{~mm}$. Para as extrações foram utilizados os solventes: acetato de etila (F Maia), $n$-hexano (F Maia) e isopropanol (JT Baker). Os padrões de heptadecanoato de metila ( $>99 \%$ de pureza) e $\beta$-caroteno ( $>99 \%$ de pureza) e derivatizante $\mathrm{BF}_{3}$-metanol foram obtidos da Sigma-Aldrich Chemical Co. Todos os solventes e reagentes utilizados neste estudo foram de grau analítico.

\subsection{Procedimentos Experimentais}

Extração sólido-líquido: A extração do óleo da polpa de macaúba foi realizada em agitador orbital (Marconi, MA 839/A), onde $5 \mathrm{~g}$ de polpa foram colocadas em Erlenmeyers com tampa de vidro $(250 \mathrm{~mL})$ juntamente com os solventes na razão 1:8 (massa de polpa:volume de solvente). A temperatura de extração foi de $40{ }^{\circ} \mathrm{C}$, agitação de $100 \mathrm{rpm}$ e tempo de 4 horas. Após o período de extração as amostras foram filtradas e o excesso de solvente, no filtrado, foi evaporado em rota evaporador (Marconi, MA120) e o remanescente mantido em estufa até peso constante. Os extratos foram armazenados sob refrigeração até a realização das análises, as quais foram realizadas em duplicata. $O$ rendimento foi calculado como a razão entre a massa extraída de óleo e a massa de polpa utilizada. 
Análises dos Extratos Obtidos: A concentração de $\beta$-caroteno nos extratos foi determinada em espectrofotômetro UV (Femto, 700 plus). As amostras foram preparadas em $n$-hexano e o teor de $\beta$-caroteno foi determinada em $450 \mathrm{~nm}$. Soluções de $\beta$-caroteno (Sigma-Aldrich) foram preparadas em concentrações de $1,0-100,0 \mathrm{mg} \mathrm{L}^{-1}$ para obter a curva de calibração com coeficiente de regressão $(\mathrm{R})$ de 0,998. A análise dos flavonóides totais foi realizada de acordo com o procedimento reportado por Francis (1982). Em um balão de $50 \mathrm{~mL}$, pesou-se $1 \mathrm{~g}$ de óleo, completou-se o volume do balão com etanol/HCl $\left(1,5 \mathrm{~mol} \mathrm{~L}^{-1}\right)$ e deixou-se a amostra descansar em local protegido da luz por 12 horas. A leitura da absorbância foi realizada em espectrofotômetro (Femto, 700plus) em $374 \mathrm{~nm}$. Com a finalidade de determinar o perfil de ácidos graxos totais presentes nas amostras de óleo extraídas por cromatografia gasosa, foi realizada a derivatização do óleo com solução metanólica de $\mathrm{BF}_{3}$ seguindo a metodologia padrão $\mathrm{AOAC} C \mathrm{Ce}$ 2-66 (Walker, 1990). Posteriormente, as análises foram conduzidas em cromatógrafo a gás acoplado a espectro de massa (Thermo-Finnigan), utilizando uma coluna capilar Agilent HP-5MS ( $30 \mathrm{~m} \times 0,250 \mathrm{~mm} \times 0,25 \mu \mathrm{m})$ e as condições utilizadas foram: injeção de $0,4 \mu \mathrm{L}$ no modo split $1: 10$, temperatura inicial da coluna de $120{ }^{\circ} \mathrm{C}$, mantida nesta temperatura por 5 minutos, aumentando para $180{ }^{\circ} \mathrm{C}$ à taxa de $15{ }^{\circ} \mathrm{C} \min ^{-1}$ e para $240{ }^{\circ} \mathrm{C}$ à $5{ }^{\circ} \mathrm{C} \mathrm{min}{ }^{-1}$, permanecendo por 5 minutos. A vazão do gás de arraste, hélio, foi de $1 \mathrm{~mL} \min ^{-1}$. A identificação dos componentes presentes nas amostras foi realizada pelo programa Xcalibur ${ }^{\circledR}$ (Thermo Electron) e para a quantificação dos ácidos graxos totais, heptadecanoato de metila foi utilizado como padrão interno.

Caracterização do Farelo Desengordurado: As análises físico-químicas foram realizadas de acordo com as metodologias da AOAC (2005). Para as análises tecnológicas, as amostras de farelo desengordurado foram secas em estufa à $60^{\circ} \mathrm{C}$ até peso constante e trituradas em moinho de facas tipo Willye Modelo SL-031. Após a moagem foram peneiradas e a fração classificada como farinha fina com 100 mesh foi destinada às análises. Foram realizadas as análises de volume de intumescimento (VI), índice de absorção de água (IAA) e índice de absorção de óleo (IAO) segundo metodologia proposta por Seibel (2006). A atividade emulsificante foi realizada conforme metodologia proposta por Dench et al. (1981).

\section{RESULTADOS E DISCUSSÃO}

\subsection{Rendimento e Caracterização dos Extratos}

Os resultados de rendimento em óleo utilizando diferentes solventes e teores de $\beta$-caroteno e flavonóides nos extratos obtidos são apresentados na Tabela 1. Os resultados obtidos demonstram maiores rendimento para a utilização do isopropanol e reporta-se que os rendimentos utilizando acetato de etila e $n$-hexano não apresentaram diferença significativa $(\mathrm{p}>0,05)$. A extração do óleo pode ser atribuída à polaridade dos solventes, o acetato de etila apresenta maior polaridade, seguido pelo isopropanol e o $n$-hexano apresenta propriedades apolares (Takeuchi et al., 2009; Ramluckan et al., 2014). Os solventes com característica apolar, como o $n$-hexano, extraem seletivamente lipídios superficiais (Ramluckan et al., 2014), já o isopropanol por ser um álcool pode apresentar características bipolares, apresentando rendimento superior em comparação a outros solventes, demonstrando-se adequado para extração de compostos bioativos (Almeida et al., 2012) e da fração lipídica (Rodriguez-Solana et al., 2014). Em outros trabalhos da literatura foi observado que o isopropanol proporcionou maior rendimento no processo de extração. Seth et al. (2007) realizaram a extração do óleo de flocos de soja comercial e relatam rendimentos de $84,2 \%$ e $77,8 \%$ utilizando como solvente o isopropanol e o $n$-hexano, respectivamente. Dutta et al. (2014) extraíram o óleo de sementes de Crotalaria juncea em Soxhlet e reportam que o isopropanol apresentou maior rendimento em óleo $11,34 \%$ quando comparado ao $n$-hexano 2,3\%. 
A polaridade não é a única o fator que afeta a eficiência de extração, sendo importante compreender as diferentes interações entre soluto e solvente (Almeida et al., 2012). Tian et al. (2013) extraíram óleo da semente de romã com $n$-hexano, acetato de etila e isopropanol utilizando ultrassom a $40^{\circ} \mathrm{C}$ em 36 minutos. $\mathrm{O} n$-hexano apresentou melhor rendimento em óleo $(20,48 \%)$ seguido pelo acetato de etila $(20,04 \%)$ e isopropanol $(18,56 \%)$. Pode ser observado na Tabela 1 que rendimentos em óleo entre 23 e $27,43 \%$ foram obtidos. Na literatura relatam-se teores de $18,7 \%$ a $29 \%$ de óleo na polpa de Acrocomia aculeata. Ciconini et al. (2013) e Oliveira et al. (2013b) utilizaram éter de petróleo na extração por Soxhlet, obtendo rendimento de 25,1\% e $18,7 \%$ em óleo, respectivamente. Coimbra e Jorge (2012) utilizaram $n$-hexano na extração por Soxhlet e reportam $28,94 \%$ de óleo.

Tabela 1 - Resultados de rendimento em óleo e teores de $\beta$-caroteno e flavonóides na polpa e extratos obtidos com diferentes solventes. Médias seguidas pela mesma letra, não se diferem estatisticamente $(\mathrm{p}>0,05)$.

\begin{tabular}{|c|c|c|c|c|}
\hline & \multicolumn{4}{|c|}{ Solvente } \\
\hline & Polpa & $\begin{array}{l}\text { Acetato de } \\
\text { Etila }\end{array}$ & n-hexano & Isopropanol \\
\hline Rendimento (\%) & $25,72 \pm 0,02^{1}$ & $22,97 \pm 0,33^{\mathrm{a}}$ & $23,39 \pm 0,15^{\mathrm{a}}$ & $27,43 \pm 0,33^{b}$ \\
\hline$\beta$-caroteno $\left(\mathrm{mg} 100 \mathrm{~g}^{-1}\right)$ & $44,15 \pm 0.06$ & $305,49 \pm 0,60^{a}$ & $331,13 \pm 0,47^{\mathrm{b}}$ & $345,46 \pm 0,31^{b}$ \\
\hline Flavonóides (mg $\left.100 \mathrm{~g}^{-1}\right)$ & $16,40 \pm 0.10$ & $13,87 \pm 0,05^{\mathrm{a}}$ & $13,61 \pm 0,05^{\mathrm{b}}$ & $14,79 \pm 0,05^{\mathrm{c}}$ \\
\hline
\end{tabular}

${ }^{1}$ Obtido pelo método de Soxhlet com éter de petróleo como solvente em refluxo por 8 horas.

Em relação aos teores de $\beta$-caroteno e flavonóides relatados na Tabela 1, verifica-se que o isopropanol forneceu um extrato com maiores concentrações de flavonóides, seguido do acetato de etila e $n$-hexano $(\mathrm{p}<0,05)$. Os flavonóides são moléculas polares, sendo extraídas por solventes com maior polaridade (Huber e Rodriguez-Amaya, 2008), como o isopropanol. Em relação aos teores de $\beta$-caroteno, o isopropanol e $n$-hexano apresentaram maior eficiência na extração deste composto, sendo que não foi observada diferença significativa $(\mathrm{p}>0,05)$ entre os solventes. Na literatura relata-se que o $n$-hexano é um bom solvente para o $\beta$-caroteno (Mustapa et al., 2011; Jaime et al., 2010; Sagani et al., 2005). Os carotenóides em geral apresentam moléculas com características apolares (Temelli et al., 2008), entretanto alcoóis podem apresentar características bipolares, dissolvendo com facilidade substâncias orgânicas apolares (Takeuchi et al., 2009), desta forma, o $\beta$-caroteno pode ser adequadamente extraídos por solventes como o isopropanol (Mezzomo et al., 2011). O acetato de etila apresentou menor rendimento na extração do $\beta$ caroteno, quando comparado aos demais solvente, no entanto, Três et al. (2007) reporta que o $\beta$ caroteno apresenta elevada solubilidade em acetato de etila.

A Tabela 2 apresenta o perfil de ácidos graxos dos extratos obtidos utilizando os diferentes solventes, onde verifica-se que, em geral, os teores não foram afetados $(p>0,05)$ pela natureza do solvente extrator. Conforme os resultados apresentados na Tabela 2 os extratos apresentaram elevados teores de ácido oleico $(\sim 60 \%)$ e ácido palmítico $(\sim 27 \%)$, e baixas concentrações do ácido linoleico $(\sim 6 \%)$ e linolênico $(\sim 3 \%)$. Alguns autores na literatura reportam resultados semelhantes para a composição do óleo de polpa da macaúba. Coimbra e Jorge (2011) obtiveram como principais ácidos graxos o ácido oleico $(52,6 \%)$ e ácido palmítico $(24,6 \%)$. Hiane et al. (2005) analisaram a composição do óleo de macaúba, obtendo predominância do ácido oleico $(62,2 \%)$ e palmítico $(18,5 \%)$ e teores de $4 \%$ do ácido linoleico. Amaral et al. (2011) reportam como principais ácidos graxos no óleo da polpa de macaúba o ácido oleico $(69,07 \%)$, ácido palmítico $(12,18 \%)$ e ácido linoléico (6,77\%). A variação da composição do óleo da Acrocomia aculeata pode estar relacionada à variabilidade fenotípicas (Ciconini et al., 2013), aspectos relacionados ao clima podem se manifestar na planta por meio de processos fisiológicos, provocando variações, na 
produção e características do óleo. Os ácidos graxos saturados, monoinsaturados e poli-insaturados apresentaram valores similares aos reportados por Hiane et al. (2005) e Coimbra e Jorge (2011b).

Tabela 2 - Quantificação de ácidos graxos do óleo da polpa de macaúba obtidos com diferentes solventes. Médias seguidas pela mesma letra, não se diferem estatisticamente $(p>0,05)$.

\begin{tabular}{|l|l|c|c|c|}
\hline Solvente & & Acetato de Etila & n-hexano & Isopropanol \\
\hline Ácidos graxos $^{1}$ & Caprílico & $0,275 \pm 0,03^{\mathrm{a}}$ & $0,136 \pm 0,01^{\mathrm{b}}$ & $0,095 \pm 0,01^{\mathrm{b}}$ \\
& Cáprico & $0,040 \pm 0,01^{\mathrm{a}}$ & $0,057 \pm 0,01^{\mathrm{a}}$ & $0,035 \pm 0,01^{\mathrm{b}}$ \\
\hline & Láurico & $0,091 \pm 0,01^{\mathrm{a}}$ & $0,111 \pm 0,01^{\mathrm{a}}$ & $0,114 \pm 0,01^{\mathrm{a}}$ \\
\hline & Mirístico & $0,220 \pm 0,01^{\mathrm{a}}$ & $0,184 \pm 0,02^{\mathrm{a}}$ & $0,183 \pm 0,01^{\mathrm{a}}$ \\
\hline & Palmítico & $27,777 \pm 0,29^{\mathrm{a}}$ & $27,395 \pm 0.12^{\mathrm{a}}$ & $27,242 \pm 0,42^{\mathrm{a}}$ \\
\hline & Esteárico & $1,809 \pm 0,29^{\mathrm{a}}$ & $1,804 \pm 0,14^{\mathrm{a}}$ & $2,488 \pm 0,46^{\mathrm{a}}$ \\
\hline & Oleico & $61,034 \pm 0,48^{\mathrm{a}}$ & $61,157 \pm 0,15^{\mathrm{a}}$ & $60,169 \pm 0,51^{\mathrm{a}}$ \\
\hline & Linoléico & $5,794 \pm 0,03^{\mathrm{a}}$ & $6,344 \pm 0,19^{\mathrm{b}}$ & $5,725 \pm 0,07^{\mathrm{a}}$ \\
\hline & Linolênico & $2,961 \pm 0,11^{\text {ab }}$ & $2,813 \pm 0,05^{\mathrm{a}}$ & $3,095 \pm 0,07^{\mathrm{b}}$ \\
\hline & & 30,212 & 29,687 & 30,157 \\
\hline AGS $^{2}$ & & 61,034 & 61,157 & 60,169 \\
\hline AGM $^{3}$ & & 8,755 & 9,157 & 8,820 \\
\hline AGP $^{4}$ & & & & \\
\hline
\end{tabular}

\subsection{Caracterização do Farelo desengordurado}

A caracterização físico-química da polpa (sem remoção do óleo) e dos farelos obtidos a partir da extração com os diferentes solventes é apresentada na Tabela 3. Pode se verificar que a remoção do óleo possibilita o aumento dos teores de proteína e fibras no farelo.

Tabela 3 - Características físico-químicas e cor da polpa de macaúba e farelo desengordurado (em base seca). Médias seguidas pela mesma letra, não se diferem estatisticamente ( $p>0,05)$.

\begin{tabular}{|l|c|c|c|c|}
\hline \multicolumn{1}{|c|}{} & \multicolumn{3}{|c|}{ Solventes } \\
\hline Cinzas & Polpa & Acetato de Etila & n-hexano & Isopropanol \\
\hline Fibra Bruta & $2.42 \pm 0.03^{\mathrm{a}}$ & $3,96 \pm 0,12^{\mathrm{b}}$ & $3,86 \pm 0,24^{\mathrm{c}}$ & $4,63 \pm 0,24^{\mathrm{b}}$ \\
\hline Lipídios & $8,76 \pm 0,50^{\mathrm{a}}$ & $12,74 \pm 0,14^{\mathrm{b}}$ & $11,41 \pm 0,15^{\mathrm{c}}$ & $9,89 \pm 0,39^{\mathrm{a}}$ \\
\hline Proteína & $26,31 \pm 0,02^{\mathrm{la}}$ & $1,80 \pm 0,20^{\mathrm{b}}$ & $1,80 \pm 0,39^{\mathrm{b}}$ & $1,30 \pm 0,13^{\mathrm{b}}$ \\
\hline & $3,79 \pm 0,07^{\mathrm{a}}$ & $6,58 \pm 0,04^{\mathrm{a}}$ & $6,71 \pm 0,03^{\mathrm{a}}$ & $6,65 \pm 0,06^{\mathrm{a}}$ \\
\hline
\end{tabular}

A Tabela 4 apresenta as características tecnológicas da polpa (sem remoção do óleo) e dos farelos desengordurados com os diferentes solventes.

Tabela 4 - Características tecnológicas da polpa de macaúba e farelo desengordurado. Médias seguidas pela mesma letra, não se diferem estatisticamente $(\mathrm{p}>0,05)$.

\begin{tabular}{|l|c|c|c|c|}
\hline & Polpa & Acetato de Etila & $n$-hexano & Isopropanol \\
\hline $\begin{array}{l}\text { Índice de absorção de água (g de } \\
\text { água/g de matéria seca) }\end{array}$ & $2,69 \pm 0,05^{\mathrm{a}}$ & $3,61 \pm 0,26^{\mathrm{b}}$ & $3,89 \pm 0,05^{\mathrm{b}}$ & $3,98 \pm 0,05^{\mathrm{b}}$ \\
\hline $\begin{array}{l}\text { Índice de absorção de óleo }(\mathrm{g} \text { de } \\
\text { óleo/g de matéria seca) }\end{array}$ & $2,25 \pm 0,39^{\mathrm{a}}$ & $2,86 \pm 0,03^{\mathrm{a}}$ & $2,88 \pm 0,05^{\mathrm{a}}$ & $3,01 \pm 0,24^{\mathrm{a}}$ \\
\hline $\begin{array}{l}\text { Volume de Intumescimento }(\mathrm{mL}) \\
\text { Capacidade Emulsificante }(\%)\end{array}$ & $3,75 \pm 0,35^{\mathrm{a}}$ & $3,75 \pm 0,35^{\mathrm{a}}$ & $4,0 \pm 0,0^{\mathrm{a}}$ & $4,75^{\mathrm{a}} \pm 0,35^{\mathrm{a}}$ \\
\hline
\end{tabular}


Pode ser observado que a remoção do óleo aumentou a capacidade de retenção de água dos farelos, porém quanto as demais características tecnológicas não foi verificada diferença. As propriedades de hidratação (IAA e VI) são importantes para utilização como ingredientes funcionais para evitar sinérese e modificar a viscosidade e textura de alimentos industrializados. Já a capacidade emulsificante e de retenção de óleo são características importantes que permitem a estabilização de produtos gordurosos e emulsificados. (Elleuch et al.,2011). Os resultados das características tecnológicas indicam que o farelo desengordurado da macaúba pode ser utilizado como um ingrediente funcional em alimentos.

\section{CONCLUSÃO}

O isopropanol apresentou maior eficiência na extração do óleo da polpa de macaúba, obtendo os maiores teores de $\beta$-caroteno e flavonóides no extrato. O perfil de ácidos graxos dos extratos, em geral, não foram afetados $(\mathrm{p}>0,05)$ pela natureza do solvente extrator, sendo os principais ácidos graxos, o ácido palmítico e o ácido oleico. O farelo desengordurado apresenta maiores teores de proteína e fibras. A remoção do óleo favorece a capacidade de retenção de água e óleo e volume de intumescimento do farelo, no entanto, não apresentou influência na capacidade emulsificante.

\section{REFERÊNCIAS BIBLIOGRÁFICAS}

AOAC - Association of official analytical chemist. Official methods of analysis. 16 th ed. Gaithersburg: AOAC, 2005.

ABREU, I. S.; CARVALHO, C. R.; CARVALHO, G. M.; MOTOIKE, S. Y. First karyotype, DNA C-value and AT/GC base composition of macaw palm (Acrocomia aculeata) - a promising plant for biodiesel production. Australian J. Botany, v.59, p.149-155, 2011.

ALMEIDA, P. P.; MEZZOMO, N.; FERREIRA, S. R. S. Extraction of Mentha spicata L. Volatile Compounds: Evaluation of Process Parameters and Extract Composition. Food Bioproc. Technol. v. 5, p.548-559, 2012.

AMARAL, F. P.; BROETTO, F.; BATISTELlA, C. B.; JORGE, S. M. A. Extração e caracterização qualitativa do óleo da polpa e amêndoas de frutos de macaúba [acrocomia aculeata (jacq) lodd. ex mart] coletada na região de Botucatu, SP. Rev. Energ. Agric., v. 26, p. 12-20, 2011.

AQUINO, L. P.; FERRUA, F. Q. BORGES, S. V.; CIRILlO, M. A.; VIEIRA, A. P. Influência do Pré-tratamento da Polpa de Pequi (Caryocar Brasiliense Camb.) no Rendimento do Extrato Lipídico. Alim. Nutr., v.20, p. 289-294, 2009.

CICONINI, G.; FAVARO, S. P.; ROSCOE, R.; MIRANDA C. H. B.; TAPETI, C. F.; MIYAHIRA, M. A. M.; BEARARI, L.; GALVANI, F.; BORSATO, A. V.; COLNAGO, L. A.; NAKA M. H. Biometry and oil contents of Acrocomia aculeata fruits from the Cerrados and Pantanal biomes in Mato Grosso do Sul, Brazil. Ind. Crops Prod., v.45, p. 208-214, 2013.

COIMBRA, M. C.; JORGE, N. Fatty acids and bioactive compounds of the pulps and kernels of Brazilian palm species, guariroba (Syagrus oleraces), jerivá (Syagrus romanzoffiana) and macaúba (Acrocomia aculeata). J Sci Food Agric., v. 92, p. 679-684, 2012.

COIMBRA, M. C.; JORGE, N. Proximate composition of guariroba (Syagrus oleracea), jerivá (Syagrus romanzoffiana) and macaúba (Acrocomia aculeata) palm fruits. Food Research Internat., v. 44, p. 2139-2142, 2011 a. 
COIMBRA, M. C.; JORGE, N. Characterization of the Pulp and Kernel Oils from Syagrus leracea, Syagrus romanzoffiana, and Acrocomia aculeata. J. Food Sci., v.76, p. 11561161, 2011b.

DENCH,J.E; RIVAS,N.; CAYGILL, J.C. Selected functional properties of sesame (Sesamum indicum L.) flour and two protein isolates. J. Sci.Food Agri., v.32,p.557-564,1981.

DUTTA, R.; SARKAR, U.; MUKHERJEE, A. Extraction of oil from Crotalaria Juncea seeds in a modified Soxhlet apparatus: Physical and chemical characterization of a prospective bio-fuel. Fuel, v. 116, p. 794-802, 2014.

ELLEUCH, M.; BEDIGIAN, D.; ROISEUX, O.; BESBES, S.; BLECKER, C.; ATTIA, H. Dietary fibre and fibre-rich by-products of food processing: Characterisation, technological functionality and commercial applications: A review. Food Chem, v.124, p.411-421, 2011.

FRANCIS, F.J. Analysis of anthocyanins. In: MARKAKIS, P. (ed). Anthocyanins as food colors. New York: Academic Pres, p.181-207, 1982.

FREITAS, S. P.; FREITAS-SILVA, O.; MIRANDA I. C.; COELHO, M. A. Z. Extração e fracionamento simultâneo do óleo da castanha-do-Brasil com etanol. Ciênc. Tecnol. Alim., v. 27, p. 14-17, 2007.

HIANE, P. A.; RAMOS FILHO, M. M.; RAMOS, M. I. L.; MACEDO, M. L. R. Bocaiúva, Acrocomia aculeate (Jacq.) Lodd., pulp and kernel oils: characterization and fatty acid composition. Braz. J. Food Technol., v. 8, p. 256-259, 2005.

HUBER, L. S.; RODRIGUEZ-AMAYA, D. B. Flavonoids and flavones the Brazilian sources and factors that influence the composition in food. Food Nutrit., v.19, p. 97-108, 2008.

JAIME, L.; RODRIGUEZ-MEIZOSO, I.; CIFUENTES, A.; SANTOYO, S.; SUAREZ, S.; IBANEZ, E.; SENORANS, F.J. Pressurized liquids as an alternative process to antioxidant carotenoids' extraction from Haematococcus pluvialis microalgae. LWT Food Sci. Technol., v. 43, p. 105-112, 2010.

MEZZOMO, N.; MAESTRIA, B.; SANTOS, R. L.; MARASCHIN, M.; FERREIRA, S. R. S. Pink shrimp (P. brasiliensis and P. paulensis) residue: Influence of extraction method on carotenoid concentration. Talanta, v. 85, p. 1383-1391, 2011.

MUSTAPA, A. N., MANAN, Z. A., AZIZI, C. Y. M., SETIANTO, W. B., OMAR, A. K. M. Extraction of b-carotenes from palm oil mesocarp using sub-critical R134a. Food Chem., v.125, p. 262-267, 2011.

OLIVEIRA, R.C.; BARROS, S.T.D.; GIMENES, M.L. The extraction of passion fruit oil with green solventes. J.Food Eng., v. 117, pp.458-463, $2013 \mathrm{a}$.

OLIVEIRA, D. M.; COSTA, J. P.; CLEMENTE, E.; COSTA, J. M. C. Characterization of Grugru Palm Pulp for Food Applications. J. Food Sci. Eng., v. 3, p.107-112, 2013 b.

OLIVEIRA, R.C.; GUEDES, T.A.; GIMENES, M.L.; BARROS, S.T.D. Effect of process variables on the oil extraction from passion fruit seeds by conventional and nonconvention techniques. Acta Scien. Tech., v. 36, p.87-91, 2014.

PIETA, P. G. Flavonoids as Antioxidants. J. Nat. Prod., v. 63, p. 1035-1042, 2000.

PRIOR, R. L.; CAO, G. Antioxidant phytochemicals in fruits and vegetables. Diet and health implication. Horticult. Sci., v.35, p.588-592, 2000. 
RAMLUCKAN, K.; MOODLEY, K. G.; BUX, F. An evaluation of the efficacy of using selected solvents for the extraction of lipids from algal biomass by the soxhlet extraction method. Fuel, v. 116, p. 103-108, 2014.

RAMOS, M. I. L.; RAMOS FILHO, M. M.; HIANE, P. A.; BRAGA NETO, J. A.; SIQUEIRA, E. M. A. Qualidade nutricional da polpa de bocaiúva Acrocomia aculeata (Jacq.) Lodd. Ciênc. Tecnol. Alim., v. 28, p. 90-94, 2008.

RODRÍGUEZ-SOLANA, R.; SALGADO, J. M.; DOMÍNGUEZ, J. M.; CORTÉSDIÉGUEZ, S. Characterization of fennel extracts and quantification of estragole: Optimization and comparison of accelerated solvent extraction and Soxhlet techniques. Ind. Crops Prod., v. 52, p. 528- 536, 2014.

SANAGI, M. M.; SEE, H. H.; IBRAHIM, W. A. W.; NAIM, A. A. Determination of carotene, tocopherols and tocotrienols in residue oil from palm pressed fiber using ressurized liquid extraction-normal phase liquid chromatography. Anal. Chim. Acta, v. 538, p. 71-76, 2005.

SANJINEZ-ARGANDOÑA, L. J.; CHUBA, C. A. M. caracterização biométrica, física e química de frutos da palmeira bocaiuva Acrocomia aculeata (Jacq) Lodd . Rev. Bras. Frutic., v. 33, n. 3, p. 1023-1028, 2011.

SEIBEL, N. F. Caracterização, Fracionamento e Hidrólise Enzimática dos Componentes do Resíduo do Processamento da Soja [Glycine Max (L.) Merrill], Fibras dos Cotilédones. Universidade Estadual de Londrina, 2006.

SETH, S.; AGRAWALA, Y. C.; GHOSHB, P. K.; JAYASB, D. S.; SINGH, B. P. N. Oil extraction rates of soya bean using isopropyl alcohol as solvent. Biosyst. Eng., v. 97, p. $209-217,2007$.

SILVA, G. C. R.; ANDRADE, M. H. C. Development and simulation of a new oil extraction process from fruit of macauba palm tree. J. Food Proc. Eng., v. 36., p. 134-145, 2013.

TAKEUCHI, T. M; PEREIRA,C. G.; BRAGA, M. E. M.; MARÓSTICA,M. R. JR.; LEAL,P. F.; MEIRELES, M. A. A. Low-Pressure Solvent Extraction (Solid-Liquid Extraction, Microwave Assisted, and Ultrasound Assisted) from Condimentary Plants. In: Meireles, M. A. A. Extracting bioactive compounds for food products: theory and applications. CRC Press, p. 137-218, 2009.

TEMELLI,F.; SALDANA,M. D. A.; MOQUIN, P. H. L.; SUN, M. Supercritical Fluid Extraction of Specialty Oils. In: Martínez, J. L. Supercritical Fluid Extraction of Nutraceuticals and Bioactive Compounds. CRC Press, p. 51-102, 2008.

TRÊS, M.V.; FRANCHESCHI, E.; BORGES, G.R.; CORAZZA, F.C.; OLIVEIRA, J.V.; CORAZZA, M.L. Influência da temperatura na solubilidade de b-caroteno em solventes orgânicos à pressão ambiente. Ciênc. Tecnol. Aliment., v.27, p.737-748, 2007

TIAN, Y.; XU, Z.; ZHENG, B.; LO, Y. M. Optimization of ultrasonic-assisted extraction of pomegranate (Punica granatum L.) seed oil. Ultrason. Sonochem., v. 20, p. 202-208, 2013.

VATAI, T.; SKERGET, M.; KNEZ, Z. Extraction of phenolic compounds from elder berry and different grape marc varieties using organic solvents and/or supercritical carbon dioxide. J. Food Eng., v. 90, p., 246-254, 2006.

WALKER, R. E. Official methods and recommended practices of the American Oil Chemists' Society (Method AOCS Ce 2-66) (4th). Champaign: American Oil Chemists Society, 1990. 